\title{
Critical Point Dominance in One Dimension
}

\author{
C. M. Newman \\ Department of Mathematics, The University of Arizona, Tucson, AZ 85721, USA
}

\begin{abstract}
The renormalized, dimensionless 4-point coupling constant of scalar one dimensional field theories is maximized uniquely by the "critical point theories" (obtainable as the scaling limit of $\phi^{4}$ models). The renormalized coupling constant of certain scalar one dimensional lattice field theories is maximized uniquely (for fixed correlation length) by the corresponding spin-1/2 model.
\end{abstract}

\section{Introduction}

For a scalar, Euclidean field, $\phi(x), x \in \mathbb{R}^{d}$, with truncated Schwinger (Ursell) functions $U_{n}\left(x_{1}, \ldots, x_{n}\right)$, and physical mass $m>0$, one definition of the renormalized, dimensionless coupling constant $g$, which is particularly appropriate for $\phi^{4}$ models (see [6]) is

$$
g=m^{d} B / A^{2}
$$

where

$$
\begin{aligned}
A & =\lim _{L \rightarrow \infty} L^{-d} \int_{\left(C_{L}\right)^{2}} U_{2}\left(x_{1}, x_{2}\right) d x_{1} d x_{2}=\int_{\mathbb{R}^{d}} U_{2}(0, x) d x, \\
B & =\lim _{L \rightarrow \infty} L^{-d} \int_{\left(C_{L}\right)^{4}}\left[-U_{4}\left(x_{1}, x_{2}, x_{3}, x_{4}\right)\right] d x_{1} d x_{2} d x_{3} d x_{4} \\
& =\int_{\left(\mathbb{R}^{d}\right)^{3}}\left[-U_{4}\left(0, y_{1}, y_{2}, y_{3}\right)\right] d y_{1} d y_{2} d y_{3},
\end{aligned}
$$

and $C_{L}$ denotes the cube, $([-L / 2, L / 2])^{d}$, in $\mathbb{R}^{d}$.

In [4], it was proven by using correlation inequalities, that in $\phi^{4}$ models, $g$ has an absolute upper bound (depending only on $d$ ) and in [3] it was argued that the value of $g$ for $\phi^{4}$ models should be dominated by its critical point value. The resulting picture of critical point dominance and its relation with renormalization group analysis is presented rather clearly in [11] (see especially Fig. 5 there) where

* $\quad$ Alfred P. Sloan Research Fellow, on leave from Indiana University. Research supported in part by NSF Grant MCS 77-20683 and by the U.S.-Israel Binational Science Foundation 
the critical point value is denoted $g_{w}$, the $w$ standing for Wilson [12]. The dependence of $g_{w}$ on $d$ and the possible existence of (non-superrenormalizable) $\phi^{4}$ models with $g>g_{w}$ are among the main topics discussed in [11]. For numerical results concerning $g_{w}(d)$, see $[1,2]$.

The primary purpose of the present paper is to show, under rather mild assumptions that at least for $d=1$, there can be no field theories (whether or not of $\phi^{4}$ type) with $g>g_{w}$, and moreover that $g=g_{w}$ only for the ( $\phi^{4}$ scaling limit) "critical point theories". These results verify in a particularly strong way the Glimm and Jaffe picture of critical point dominance for $d=1$.

The scaling limit theories, which have been extensively studied by Glimm and Jaffe [5], have strictly positive physical mass $\bar{m}$, and are believed to be obtainable by either of two (presumably equivalent - at least for low dimension [1]) limiting procedures. These procedures automatically involve approaching the critical point so that for the scaling limits, one expects to have $g=g_{w}$. In the first procedure one lets $\lambda$ tend to $\infty$ in a $\lambda \phi^{4}$ model while varying the bare mass so that the physical mass tends to $\bar{m}$; in the second procedure one lets the lattice spacing $\varepsilon$ tend to 0 in a spin- $1 / 2$ Ising model while varying the temperature so that the physical correlation length has the finite limit, $1 / \bar{m}$. Our critical point theories will be parametrized by the physical mass $\bar{m}$ (replaced by $m$ in Example 3 below) and a field scaling variable (the $K$ of Examples 2 and 3 ). In order to explicitly define these one dimensional scaling limits, we first define what we will mean in this paper by one dimensional field theories in general. We replace $x$ by $t$ or $s$ since the one dimensional variable is Euclidean time; we also write $\operatorname{spec}(T)$ [respectively $\operatorname{Dom}(T)]$ for the spectrum (respectively domain) of an operator $T$ and denote by $\Omega^{\perp}$ the orthogonal complement of the subspace generated by $\Omega$.

Definition 1. A one dimensional (continuum) field theory of physical mass $m>0$, consists of a Hilbert space $\mathscr{H}$, a self-adjoint operator $H \geqq 0$, and its related semigroup $P^{s} \equiv \exp (-s H)$, a unit vector $\Omega \in \mathscr{H}$, and a self-adjoint operator $\Phi$ such that

(i) $P^{1} \Omega=\Omega$,

(ii) $\sup \left\{\operatorname{spec}\left(Q P^{1} Q\right)\right\}=e^{-m}$ where $Q$ is the projection on $\Omega^{\perp}$ [i.e., $Q \Psi=\Psi$ $-(\Omega, \Psi) \Omega]$,

(iii) for $n=0,1,2, \ldots$ and for any choice of non-negative $s_{1}, \ldots, s_{n}$, the vector

$$
\Psi\left(s_{1}, \ldots, s_{n}\right) \equiv P^{s_{1}} \Phi \ldots P^{s_{n}} \Phi \Omega,
$$

belongs to $\operatorname{Dom}(\Phi)$, and

(iv) finite linear combinations of the $\Psi\left(s_{1}, \ldots, s_{n}\right)$ 's are dense in $\mathscr{H}$. The Schwinger functions of the field theory are defined by

$$
S_{n}\left(t_{1}, \ldots, t_{n}\right)=\left(\Omega, \Psi\left(0, t_{\pi_{2}}-t_{\pi_{1}}, \ldots, t_{\pi_{n}}-t_{\pi_{n-1}}\right),\right.
$$

where $\pi$ is any permutation of $1, \ldots, n$ such that $t_{\pi_{1}} \leqq t_{\pi_{2}} \leqq \ldots \leqq t_{\pi_{n}}$.

Example 1. Even $\phi^{4}$ models: Here $\mathscr{H}=L^{2}(\mathbb{R}, d \phi), \Phi$ is multiplication by $\phi$, and

$$
H=\eta\left(-d^{2} / d \phi^{2}+\lambda\left(\phi^{2}-K\right)^{2}-E\right)
$$

for $\lambda>0$ and real $K . E=E(\lambda, K)$ is chosen so that zero is the lowest eigenvalue of $H$ and $\eta=\eta(\lambda, K, m)>0$ is chosen so that the next lowest eigenvalue is $m$. 
Definition 2. A one dimensional lattice field theory with lattice spacing $\varepsilon>0$ and physical correlation length $\xi$ consists of $\mathscr{H}, \Omega, \Phi$ as above together with a selfadjoint operator $P$ satisfying $0<P \leqq 1$ and its related discrete semigroup $P^{n}$ such that (i)-(iv) are valid with $m=\varepsilon / \xi$. The Schwinger functions $S_{n}\left(t_{1}, \ldots, t_{n}\right)$ are defined for $\left(t_{1}, \ldots, t_{n}\right) \in \varepsilon \mathbb{Z}^{d}$ by

$$
S_{n}\left(\varepsilon i_{1}, \ldots, \varepsilon i_{n}\right)=\left(\Omega, \Psi\left(0, i_{\pi_{2}}-i_{\pi_{1}}, \ldots, i_{\pi_{n}}-i_{\pi_{n-1}}\right)\right),
$$

where $\pi$ is any permutation such that $i_{\pi_{1}} \leqq i_{\pi_{2}} \ldots \leqq i_{\pi_{n}}$.

Example 2. Spin-1/2 Ising model (zero external field, $\pm \sqrt{K}$ valued): Here $\mathscr{H} \ldots \mathbb{C}^{2}$,

$$
\Phi=\sqrt{K}\left[\begin{array}{rr}
+1 & 0 \\
0 & -1
\end{array}\right]
$$

for some $K>0$, and

$$
P=\left(e^{\beta}+e^{-\beta}\right)^{-1}\left[\begin{array}{ll}
e^{\beta} & e^{-\beta} \\
e^{-\beta} & e^{\beta}
\end{array}\right]
$$

with

$$
\beta=\operatorname{arctanh}\left(e^{-m}\right)=\operatorname{arctanh}\left(e^{-\varepsilon / \xi}\right) .
$$

The scaling limit may be obtained from Example 2 by letting $\varepsilon \rightarrow 0$ and $\beta \rightarrow \infty$ with $K$ fixed (thus approaching the one dimensional "critical point" at $\beta=\infty$ ) in such a way that $\varepsilon \exp (4 \beta) \rightarrow 1 / \bar{m}$ (or equivalently $m / \varepsilon \rightarrow \bar{m}$ ). The resulting continuum field theory is our next example.

We note that the spin-1/2 Ising model is a simple two state Markov chain and its scaling limit is just the related continuous time two state Markov jump process.

Example 3. Critical point theories: Here $\mathscr{H}=\mathbb{C}^{2}, \Phi$ is as in (7), and (with $\bar{m}$ replaced by $m$ )

$$
H=\frac{1}{2}\left[\begin{array}{rr}
m & -m \\
-m & m
\end{array}\right]
$$

A unitarily equivalent theory can be obtained from Example 1 by letting $\lambda \rightarrow \infty$ with $K>0$ fixed. To appreciate the equivalence, note that in Example 1, one can use a standard procedure to replace $L^{2}(\mathbb{R}, d \phi)$ by $L^{2}\left(\mathbb{R}, \Omega^{2}(\phi) d \phi\right)$ which in the limit $\lambda \rightarrow \infty$ becomes

$$
L^{2}(\mathbb{R},[\delta(\phi-\sqrt{K})+\delta(\phi+\sqrt{K})] / 2) \cong \mathbb{C}^{2} ;
$$

see [9] for a rigorous analysis.

We will always assume the symmetry $S_{1} \equiv 0$, which can in any case be obtained by replacing $\Phi$ by $\Phi-(\Omega, \Phi \Omega)$. When $S_{1} \equiv 0$, the Ursell functions appearing in (2) and (3) are given by

$$
\begin{aligned}
U_{2}\left(x_{1}, x_{2}\right)= & S_{2}\left(x_{1}, x_{2}\right), \\
U_{4}\left(x_{1}, x_{2}, x_{3}, x_{4}\right)= & S_{4}\left(x_{1}, x_{2}, x_{3}, x_{4}\right)-S_{2}\left(x_{1}, x_{2}\right) S_{2}\left(x_{3}, x_{4}\right) \\
& -S_{2}\left(x_{1}, x_{3}\right) S_{2}\left(x_{2}, x_{4}\right)-S_{2}\left(x_{1}, x_{4}\right) S_{2}\left(x_{2}, x_{3}\right) .
\end{aligned}
$$


In Sect. 2 of this paper we treat critical point dominance for the continuum case and in Sect. 3 we treat the lattice case. The results in the two sections are analogous except that in the discrete case we find it technically necessary to make the additional assumption that

$$
S_{4}\left(t_{1}, t_{2}, t_{3}, t_{4}\right) \geqq S_{2}\left(t_{1}, t_{2}\right) S_{2}\left(t_{3}, t_{4}\right) \text { for all } t_{1} \leqq t_{2} \leqq t_{3} \leqq t_{4} ;
$$

this is a special case of the GKS inequalities $[7,10]$ and is valid for many models including lattice $\phi^{4}$ models [8]. The proofs of our results are elementary; they are related to the methods of [4] and are based on the observations that in both Examples 2 and 3, the inequality (13) is an equality and $S_{2}(0, s)=K \exp (-m s)$.

\section{Continuum Theories}

Theorem 1. Let $g$ be the renormalized coupling constant defined by (1)-(3) and (11)(12), for a one dimensional field theory of physical mass $m>0$ as given in Definition 1 above with $S_{1}(x) \equiv(\Omega, \Phi \Omega)=0$; then $g \leqq 6$ and $g=6$ if and only if the field theory is unitarily equivalent to a critical point theory of Example 3 above (for some $K>0$ ).

Proof. We let $R=Q H^{-1} Q$ where $Q$ is the projection on $\Omega^{\perp}$ and define for any operator $T,\langle T\rangle=(\Phi \Omega, T(\Phi \Omega))$. Then

$$
A=2 \int_{0}^{\infty} S_{2}(0, s) d s=2 \int_{0}^{\infty}\langle\exp (-s H)\rangle d s=2\langle R\rangle,
$$

where we have used the fact that $Q \Phi \Omega=\Phi \Omega$ since $(\Omega, \Phi \Omega)=0$. Similarly, we have

$$
B=4 ! \int_{0}^{\infty} \int_{0}^{\infty} \int_{0}^{\infty}\left[-U_{4}\left(0, s_{1}, s_{1}+s_{2}, s_{1}+s_{2}+s_{3}\right)\right] d s_{1} d s_{2} d s_{3}=B_{1}-B_{2}
$$

with

$$
\begin{aligned}
B_{1}= & 4 ! \int_{0}^{\infty} \int_{0}^{\infty} \int_{0}^{\infty}\left[S_{2}\left(0, s_{1}+s_{2}\right) S_{2}\left(0, s_{2}+s_{3}\right)\right. \\
& \left.+S_{2}\left(0, s_{1}+s_{2}+s_{3}\right) S_{2}\left(0, s_{2}\right)\right] d s_{1} d s_{2} d s_{3}, \\
B_{2}= & 4 ! \int_{0}^{\infty} \int_{0}^{\infty} \int_{0}^{\infty}\left[S_{4}\left(0, s_{1}, s_{1}+s_{2}, s_{1}+s_{2}+s_{3}\right)-S_{2}\left(0, s_{1}\right) S_{2}\left(0, s_{3}\right)\right] d s_{1} d s_{2} d s_{3} .
\end{aligned}
$$

We first perform the $s_{1}$ and $s_{3}$ integrals and then the $s_{2}$ integral to obtain

$$
\begin{aligned}
B_{1} & =4 ! \int_{0}^{\infty}\left[\left\langle\mathrm{Re}^{-s H}\right\rangle\left\langle\mathrm{Re}^{-s H}\right\rangle+\left\langle R^{2} e^{-s H}\right\rangle\left\langle e^{-s H}\right\rangle\right] d s \\
& =4 ! \int_{0}^{\infty}\left[-\frac{d}{d s}\left\{\left\langle R^{2} e^{-s H}\right\rangle\left\langle\mathrm{Re}^{-s H}\right\rangle\right\}\right] d s=4 !\left\langle R^{2}\right\rangle\langle R\rangle, \\
B_{2} & =4 ! \int_{0}^{\infty}\left[\left\langle R \Phi e^{-s H} \Phi R\right\rangle-\langle R\rangle\langle R\rangle\right] d s \\
& =4 ! \int_{0}^{\infty}\left\langle R \Phi Q e^{-s H} Q \Phi R\right\rangle d s=4 !\langle R \Phi R \Phi R\rangle .
\end{aligned}
$$


We thus have

$$
g=6 m \frac{\left\langle R^{2}\right\rangle\langle R\rangle-\langle R \Phi R \Phi R\rangle}{\langle R\rangle^{2}} .
$$

Now since $H^{-1}>0$ on $\Omega^{\perp}$, we see that

$$
\langle R \Phi R \Phi R\rangle=\left(Q[\Phi R \Phi \Omega], H^{-1} Q[\Phi R \Phi \Omega]\right) \geqq 0
$$

with equality if and only if $Q[\Phi R \Phi \Omega]=0$ or equivalently if

$$
\Phi R \Phi \Omega=\bar{K} \Omega,
$$

with $\bar{K}=(\Omega, \Phi R \Phi \Omega)=\left([\Phi \Omega], H^{-1}[\Phi \Omega]\right)>0$. We define $\Lambda$ to be the unit vector

$$
\Lambda=\left(R^{1 / 2} \Phi \Omega\right) /\left\|R^{1 / 2} \Phi \Omega\right\|=\left(R^{1 / 2} \Phi \Omega\right) /\langle R\rangle^{1 / 2},
$$

so that by $(21)$ and the fact that $m R=Q(H / m)^{-1} Q \leqq 1$, we have

$$
g \leqq 6 m \frac{\left\langle R^{2}\right\rangle}{\langle R\rangle}=6(\Lambda,(m R) \Lambda) \leqq 6,
$$

with the final inequality an equality if and only if $R \Lambda=(1 / m) \Lambda$ or equivalently if

$$
H(\Phi \Omega)=m(\Phi \Omega) .
$$

We have thus shown that $g \leqq 6$ with equality if and only if both (22) and (23) are valid. Letting $K=m \bar{K}$ and taking as basis vectors, $\left(\Omega \pm \Phi \Omega / K^{1 / 2}\right) / \sqrt{2}$, it is immediate [using property (iv) of Definition 1] that (22) and (23) are valid if and only if there is unitary equivalence with the model of Example 3 . This completes the proof.

\section{Lattice Theories}

The definition of $g$ we will use for one dimensional lattice theories is

$$
g=\varepsilon^{-1} \cdot 2 \tanh (\varepsilon / 2 \xi) \cdot B / A^{2},
$$

where $A$ and $B$ are given as in (2)-(3) but with $x_{j}$ replaced by $t_{j}=\varepsilon i_{j}$ and $\int_{C} d x$ replaced by $\varepsilon$ times the sum over all $i$ such that $\varepsilon i \in C$. Note that as $\varepsilon \rightarrow 0$ for fixed $\xi$, $\varepsilon^{-1} 2 \tanh (\varepsilon / 2 \xi)$ tends to $\xi^{-1}$ so that $(24)$ is consistent with (1).

Our main result in the lattice case [under the additional assumption of (13)], is that for fixed $\varepsilon$ and $\xi, g$ is maximized (uniquely) by the corresponding spin-1/2 model of Example 2. This is analogous to our continuum case result in that Example 2 can be obtained as the infinite coupling constant limit of lattice $\phi^{4}$ models. We note that the choice of factors in (24) implies that the $g$ of Example 2, [given by the right hand side of (25) below] is strictly bounded by its $\varepsilon \rightarrow 0$ for fixed $\xi$ value of 6 which is of course the value attained in Example 3; we also note that in Example 2, $\tanh (\varepsilon / 2 \xi)=\exp (-2 \beta)$. 
Theorem 2. Let $g$ be the renormalized coupling constant defined by (24) for a one dimensional lattice field theory of lattice spacing $\varepsilon>0$ and physical correlation length $\xi \in(0, \infty)$ as given in Definition 2 above with $S_{1}(\varepsilon i) \equiv(\Omega, \Phi \Omega)=0$ and assume that $(13)$ is valid; then

$$
g \leqq 6-2 \tanh ^{2}(\varepsilon / 2 \xi)
$$

with equality in (25) if and only if the field theory is unitarily equivalent to that of Example 2 above for some $K>0$.

Proof. For fixed $\mathscr{H}, \Omega, \Phi$, and $P$, the value of $g$ as defined by (24) is independent of $\varepsilon$; we may thus choose $\varepsilon=1$ and recall that

$$
\exp (-m) \equiv \exp (-\varepsilon / \xi)=\sup \{\operatorname{spec}(Q P Q)\} .
$$

The lattice version of Eqs. (14) and (15) is complicated by the introduction of boundary terms which vanish in the continuum case. The role played by $\int_{0}^{\infty} P^{t} d t=H^{-1}$ in the proof of Theorem 1 is replaced here by

$$
P^{0} / 2+\sum_{j=1}^{\infty} P^{j}=(1+P) / 2(1-P)
$$

and we accordingly define $R$ in this case as

$$
R=Q[(1+P) / 2(1-P)] Q
$$

note that

$$
\sup \{\operatorname{spec}(R)\}=\frac{1+e^{-m}}{2\left(1-e^{-m}\right)}=[2 \tanh (1 / 2 \xi)]^{-1} .
$$

We again define $\langle T\rangle=(\Phi \Omega, T(\Phi \Omega))$ and proceed to calculate $A$ and $B$.

$$
A=\sum_{j=-\infty}^{\infty} S_{2}(0,|j|)=\sum_{j=0}^{\infty} \varrho(j)\left\langle P^{j}\right\rangle=\langle(1+P) /(1-P)\rangle=2\langle R\rangle,
$$

where

$$
\begin{gathered}
\varrho(j)=\left\{\begin{array}{lll}
2, & \text { if } & j>0 \\
1, & \text { if } & j=0,
\end{array}\right. \\
B=\sum_{j_{1}=0}^{\infty} \sum_{j_{2}=0}^{\infty} \sum_{j_{3}=0}^{\infty} v\left(j_{1}, j_{2}, j_{3}\right)\left[-U_{4}\left(0, j_{1}, j_{1}+j_{2}, j_{1}+j_{2}+j_{3}\right)\right]=B_{1}-B_{2},
\end{gathered}
$$

where

$$
v\left(j_{1}, j_{2}, j_{3}\right)=\left\{\begin{array}{l}
4 !, \text { if no } j_{i}=0 \\
2 \cdot 3 !, \text { if a single } j_{i}=0 \\
2 \cdot 3, \text { if } j_{1}=j_{3}=0 \text { and } j_{2}>0 \\
4, \text { if } j_{1}>0 \text { and } j_{2}=j_{3}=0 \text { or if } j_{3}>0 \text { and } j_{1}=j_{2}=0 \\
1, \text { if } j_{1}=j_{2}=j_{3}=0,
\end{array}\right.
$$


and where $B_{1}$ (respectively $B_{2}$ ) is defined by a sum as in (29) but with $-U_{4}$ replaced by the integrand of (16) [respectively (17)]. We may rewrite (29) as

$$
\begin{aligned}
B= & \sum_{j_{1}=0}^{\infty} \sum_{j_{3}=0}^{\infty} \sum_{j_{2}=0}^{\infty} 6 \varrho\left(j_{1}\right) \varrho\left(j_{3}\right)\left[-U_{4}\left(0, j_{1}, j_{1}+j_{2}, j_{1}+j_{2}+j_{3}\right)\right] \\
& -\sum_{j_{1}=0}^{\infty} \sum_{j_{3}=0}^{\infty} \frac{\left(4+\varrho\left(j_{1}\right)\right) \varrho\left(j_{3}\right)+\varrho\left(j_{1}\right)\left(4+\varrho\left(j_{3}\right)\right)}{2}\left[-U_{4}\left(0, j_{1}, j_{1}, j_{1}+j_{3}\right)\right](30)
\end{aligned}
$$

with similar expressions for $B_{1}$ and $B_{2}$. In the case of $B_{1}$, we can sum over $j_{1}$ and $j_{3}$ (as in (28)) to obtain

$$
\begin{aligned}
B_{1} & =\bar{B}_{1}-\langle(5+P) /(1-P)\rangle\langle(1+P) /(1-P)\rangle-\left\langle(5+P)(1+P) /(1-P)^{2}\right\rangle\langle 1\rangle \\
& =\bar{B}_{1}-\langle 2+6 R\rangle\langle 2 R\rangle-\langle(2+6 R) 2 R\rangle\langle 1\rangle,
\end{aligned}
$$

where

$$
\begin{aligned}
\bar{B}_{1}= & \sum_{j=0}^{\infty} 6\left[\left\langle P^{j}(1+P) /(1-P)\right\rangle^{2}+\left\langle P^{j}(1+P)^{2} /(1-P)^{2}\right\rangle\left\langle P^{j}\right\rangle\right] \\
= & 6 \sum_{j=0}^{\infty}\left[\left\langle(1-P) P^{j}(1+P) /(1-P)^{2}\right\rangle\left\langle(1+P) P^{j} /(1-P)\right\rangle\right. \\
& \left.+\left\langle(1+P) P^{j}(1+P) /(1-P)^{2}\right\rangle\left\langle(1-P) P^{j} /(1-P)\right\rangle\right] \\
= & 6 \sum_{j=0}^{\infty} 2\left[\left\langle P^{j}(1+P) /(1-P)^{2}\right\rangle\left\langle P^{j} /(1-P)\right\rangle\right. \\
& -\left\langle P^{j+1}(1+P) /(1-P)^{2}\right\rangle\left\langle P^{j+1} /(1-P)\right\rangle \\
= & 12\left\langle(1+P) /(1-P)^{2}\right\rangle\langle 1 /(1-P)\rangle=12\langle 2 R(1+2 R) / 2\rangle\langle(1+2 R) / 2\rangle,
\end{aligned}
$$

so that

$$
B_{1}=24\left\langle R^{2}\right\rangle\langle R\rangle-2\langle R\rangle\langle 1\rangle .
$$

Now, by (13) and the expression for $B_{2}$ analogous to the sum of (29), we see that $B_{2} \geqq 0$ with equality if and only if

$$
\begin{gathered}
S_{4}\left(0, j_{1}, j_{1}+j_{2}+j_{3}\right)-S_{2}\left(0, j_{1}\right) S_{2}\left(0, j_{3}\right)=0 \\
\text { for all } j_{1}, j_{2}, j_{3} \geqq 0,
\end{gathered}
$$

but this is equivalent to

$$
\left\langle P^{j_{1}} \Phi Q P^{j_{2}} Q \Phi P^{j_{3}}\right\rangle=0 \text { for all } j_{1}, j_{2}, j_{3} \geqq 0,
$$

which is equivalent to

$$
Q \Phi P^{j} \Phi \Omega=0 \quad \text { for all } j \geqq 0 .
$$

Letting $\Lambda=\left(R^{1 / 2} \Phi \Omega\right) /\left\|R^{1 / 2} \Phi \Omega\right\|$ and $r=[2 \tanh (1 / 2 \xi)]^{-1}$, we see from (28) and (32) that

$$
g \leqq r^{-1} B_{1} / A^{2}=6(\Lambda,(R / r) \Lambda)-\left(2 r^{2}\right)^{-1}\left(\Lambda,(R / r)^{-1} \Lambda\right) \leqq 6-\left(2 r^{2}\right)^{-1},
$$


where the last inequality follows from (27) and is an equality if and only if $R \Lambda=r \Lambda$ or equivalently if

$$
P \Phi \Omega=e^{-m} \Phi \Omega
$$

We have thus derived (25) and seen that there is equality in (25) if and only if (34) and (36) are both valid. But (34) and (36) are equivalent to (36) together with

$$
\Phi^{2} \Omega=K \Omega \text { for some } K>0 \text {. }
$$

It is an elementary excercise, as in the proof of Theorem 1, to show that (36) and (37) are valid if and only if there is unitary equivalence with the spin-1/2 model of Example 2. This completes the proof.

Remark. One can obtain the exact expression for $B_{2}$,

$$
B_{2}=24\langle R \Phi R \Phi R\rangle-2\langle R \Phi Q \Phi\rangle-2\langle\Phi Q \Phi R\rangle,
$$

which yields the exact expression for $g$,

$$
\begin{gathered}
g=r^{-1}\langle R\rangle^{-2}\left\{6\left[\left\langle R^{2}\right\rangle\langle R\rangle-\langle R \Phi R \Phi R\rangle\right]-(1 / 2)[\langle R\rangle\langle 1\rangle-\langle R \Phi Q \Phi\rangle\right. \\
-\langle\Phi Q \Phi R\rangle]\} .
\end{gathered}
$$

It may be possible to utilize these expressions to obtain (25) without the ad hoc assumption of (13); we have been unable to do so.

Acknowledgements. The results of this paper were initially motivated by a discussion with A. Wightman concerning the ideas presented in [11]. The author would like to thank G. Baker, J.Glimm, D. Isaacson, D. Sharp, A. Sokal, and A. Wightman for useful discussions and correspondence.

\section{References}

1. Baker, G.A., Jr., Kincaid, J.M.: The continuous-spin Ising model, $g_{0}: \phi^{4}:_{d}$ field theory, and the renormalization group. J. Stat. Phys. (1981) (to appear)

2. Bender, C.M. et al.: Effective potential for a renormalized $d$-dimensional $g \phi^{4}$ field theory in the $g \rightarrow \infty$ limit. Phys. Rev. Lett. 45, 501-504 (1980)

3. Glimm, J., Jaffe, A.: Ann. Inst. Henri Poincaré 21, 27-41 (1974)

4. Glimm, J., Jaffe, A.: Ann. Inst. Henri Poincaré 22, 97-107 (1975)

5. Glimm, J., Jaffe, A. : Critical problems in quantum fields. In: Mathematical problems in theoretical physics. Dell'Antonio, G., Doplicher, S., Jona-Lasinio, G. (eds.). Berlin, Heidelberg, New York: Springer 1978

6. Glimm, J., Jaffe, A.: The coupling constant in a $\phi^{4}$ field theory. Proceedings of the 1979 Cargèse Summer School, Plenum Press (to appear)

7. Griffiths, R.B.: J. Math. Phys. (N.Y.) 8, 484-489 (1967)

8. Guerra, F., Rosen, L., Simon, B.: Ann. Math. 101, 111-259 (1975)

9. Isaacson, D.: Commun. Math. Phys. 53, 257-275 (1977)

10. Kelly, D.G., Sherman, S.: J. Math. Phys. (N.Y.) 9, 466-484 (1968)

11. Wightman, A.S.: How to parametrize the solutions of Lagrangian field theories: symmetry breaking, and dimensional interpolation and renormalization in the $\left(\phi^{4}\right)_{n}$ model. In: New developments in quantum field theory and statistical mechanics. Lévy, M., Mitter, P. (eds.). New York: Plenum Press 1977

12. Wilson, K.G. : Phys. Rev. D 7, 2911-2926 (1973)

Communicated by A. Jaffe

Received August 28, 1980 